INSTITUT NATIONAL DE RECHERCHE EN INFORMATIQUE ET EN AUTOMATIQUE

\title{
Directed Virtual Path Layouts in
} ATM networks

Jean-Claude Bermond — Nausica Marlin — David Peleg — Stéphane Pérennès

$\mathbf{N}^{\circ} 3665$

Avril 1999

THÈME 1

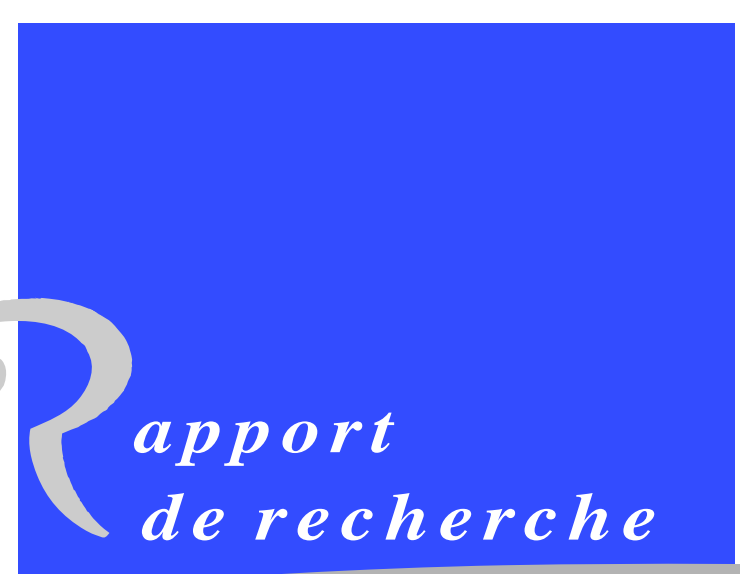





\title{
Directed Virtual Path Layouts in ATM networks
}

\author{
Jean-Claude Bermond ${ }^{*}$, Nausica Marlin ${ }^{\dagger}$, David Peleg ${ }^{\ddagger}$, Stéphane \\ Pérennès $\S$
}

Thème 1 - Réseaux et systèmes Projet Sloop

Rapport de recherche $\mathrm{n}^{\circ} 3665$ - Avril $1999-29$ pages

\begin{abstract}
Motivated by Asynchronous Transfer Mode (ATM) in telecommunication networks, we investigate the problem of designing a directed virtual topology on a directed physical topology, which consists in finding a set of directed virtual paths (VPs) satisfying some constraints in terms of load (the number of VPs sharing a physical link) and hop count (the number of VPs used to establish a connection). For both general and particular networks, such as paths, cycles, meshes, tori and trees, we derive tight bounds on the virtual diameter (the maximum hop count for a connection) as a function of the network capacity (the maximum load of a physical link).
\end{abstract}

Key-words: Graphs, ATM Networks, Virtual Path Layout, Diameter, Embedding, Load

This work has been supported by the French-Israeli cooperation "AFIRST"

* I3S Université de Nice, SLOOP joint project CNRS-UNSA-INRIA, 2004 route des Lucioles, BP93, F-06902 Sophia-Antipolis, France. (Jean-Claude.Bermond@sophia.inria.fr)

$\dagger$ I3S Université de Nice, SLOOP joint project CNRS-UNSA-INRIA, 2004 route des Lucioles, BP93, F-06902 Sophia-Antipolis, France. (Nausica.Marlin@sophia.inria.fr)

$\ddagger$ Department of Applied Mathematics and Computer Science, The Weizmann Institute, Rehovot 76100, Israel. (peleg@wisdom.weizmann.ac.il)

$\S$ I3S Université de Nice, SLOOP joint project CNRS-UNSA-INRIA, 2004 route des Lucioles, BP93, F-06902 Sophia-Antipolis, France. (Stephane.Perennes@sophia.inria.fr) 


\section{Positionnement des Chemins Virtuels dans \\ les Réseaux ATM}

Résumé : Comment construire sur un réseau physique orienté un réseau virtuel de topologie optimale au sens de diamètre et charge minimals ? Cette question est motivée par les problèmes de routage dans les réseaux ATM. Nous y répondons - pour les réseaux de topologie classique tels que le cycle, le chemin, les grilles, les tores ou les arbres - en construisant un ensemble de chemins virtuels (VPs) satisfaisant certaines contraintes de charge et de nombre de sauts. Nous donnons ainsi des bornes sur le diamètre du graphe virtuel en fonction de la capacité des liens physiques.

Mots-clés : Graphes, Réseaux ATM, Virtual Path Layout, Diamètre, Plongement, Charge 


\section{Introduction}

The advent of fiber optic media has changed the classical views on the role and structure of digital communication networks. Specifically, the sharp distinction between telephone networks, cable television networks, and computer networks has been replaced by a unified approach. The most prevalent solution for this new network challenge is Asynchronous Transfer Mode (ATM for short), which is thoroughly described in the literature [6, 15]. The transfer of data in ATM is based on packets of fixed length, termed cells. Each cell is routed independently, based on two routing fields at the cell header, called virtual channel identifier (VCI) and virtual path identifier (VPI). This method effectively creates two types of predetermined simple routes in the network, namely, routes which are based on VPIs (called virtual paths or VPs) and routes based on VCIs and VPIs (called virtual channels or VCs). VCs are used for connecting network users (e.g., a telephone call); VPs are used for simplifying network management - routing of VCs in particular. Thus the route of a VC may be viewed as a concatenation of complete VPs.

A major problem in this framework is the one of defining the set of VPs in such a way that some good properties are achieved.

1. A capacity (or bandwidth) is assigned to each VP. The sum of the capacities of the VPs that share a physical link constitutes the load of this link. Naturally, this load must not exceed the link's capacity, namely, the amount of data it can carry. The sum of the capacities of all the physical links is a major component in the cost of the network, and should be kept as low as possible.

2. The maximum number of VPs in a virtual channel, termed hop count in the literature, should also be kept as low as possible so as to guarantee low set up times for the virtual channels and high data transfer rates.

In its most general formulation, the Virtual Path Layout (VPL) problem is an optimization problem in which, given a certain communication demand between pairs of nodes and constraints on the maximum load and hop count, it is first required to design a system of virtual paths satisfying the constraints and then minimizing some given function of the load and hop count.

We employ a restricted model similar to the one presented by Cidon, Gerstel and Zaks in [12]. In particular, we assume that all VPs have equal capacities, normalized to 1 . Hence the load of a physical link is simply the number of VPs that share this link.

Although links based on optical fibers and cables are directed, traditional research uses an undirected model. Indeed, this model imposes the requirement that if there exists a VP from $u$ to $v$ then there exists also a VP from $v$ to $u$. In fact, that is the way ATM networks are implemented at the present time. However, the two VPs (the one from $u$ to $v$ and the one in the other direction) do not need to have the same capacity. Indeed, in many applications the flows on the VPs are not equal. For example, in a video application where $u$ is a server and $v$ a consumer there is a VP from $u$ to $v$ using a large capacity (transmission of video data) and a VP from $v$ to $u$ used only for control or acknowledgments with a very small 
capacity which can be considered as negligible. Therefore, it seems more reasonable to use a directed model like the one introduced by Chanas and Goldschmidt in [5]. This would allow us to model the situation described above by a single VP of capacity 1 in the main direction.

We focus on the all-to-all problem (all pairs of nodes are equally likely to communicate). Thus, the resulting maximum hop count can be viewed as the diameter of the graph induced by the VPs.

More formally, given a communication network, the VPs form a virtual directed graph (digraph) on the top of the physical one, with the same set of vertices but with a different set of arcs. (Specifically, a VP from $u$ to $v$ is represented by an arc from $u$ to $v$ in the virtual digraph.) This virtual digraph provides a Directed Virtual Path Layout (DVPL) for the physical graph. Each VC can be viewed as a simple dipath in the virtual digraph. Therefore, a central problem is to find a tradeoff between the maximum load and the virtual diameter. In this article, we consider the following problem:

Given a capacity on each physical arc, minimize the diameter of an admissible virtual graph (a virtual digraph that doesn't load an arc more than its capacity)

Related Work The problem has been considered in the undirected case, for example, in $[12,11,20,10,16,8]$. The problem of minimizing the maximum load over all VPL with bounded hop-count is studied in [9, 1], and minimizing also the average load is considered in [11]. The one-to-many problem is handled in $[9,11]$, where the focus is on minimizing the eccentricity of the virtual graph from a special point called the root (this problem is the rooted virtual path layout problem) rather than minimizing the diameter of the virtual graph. A duality in the chain network between the problem of minimizing the hop-count knowing the maximum load, and the one of minimizing the load, knowing the maximum hop-count, is established in [9]. The reader can find an excellent survey of the results in the undirected model in [21].

The techniques involved in our constructions bear a certain resemblance to various embedding techniques used previously in the context of parallel computing, in order to implement a useful virtual architecture on a given practical machine topology (cf. $[18,14])$. The parameters of interest in such embeddings are the number of virtual processors mapped onto any physical processor, the load on the physical links, and the dilation of the embedding, namely, the maximum length of the physical path corresponding to a given virtual link. The relevant concerns in our context are somewhat different, as dilation is of no consequence, and on the other hand, we have the freedom of designing the virtual topology as desired, in order to optimize its diameter.

Our Results The following table summarizes our results, giving lower and upper bounds on the virtual diameter (the minimum diameter of an admissible virtual digraph) as a function of the number of vertices $n$ in the physical graph, its diameter $D_{G}$, its maximum in- and out-degree $d$, and the capacity $c$ considered as a constant. The results mentioned for the path in the special case $c=1$ are due to $[3,4]$. 


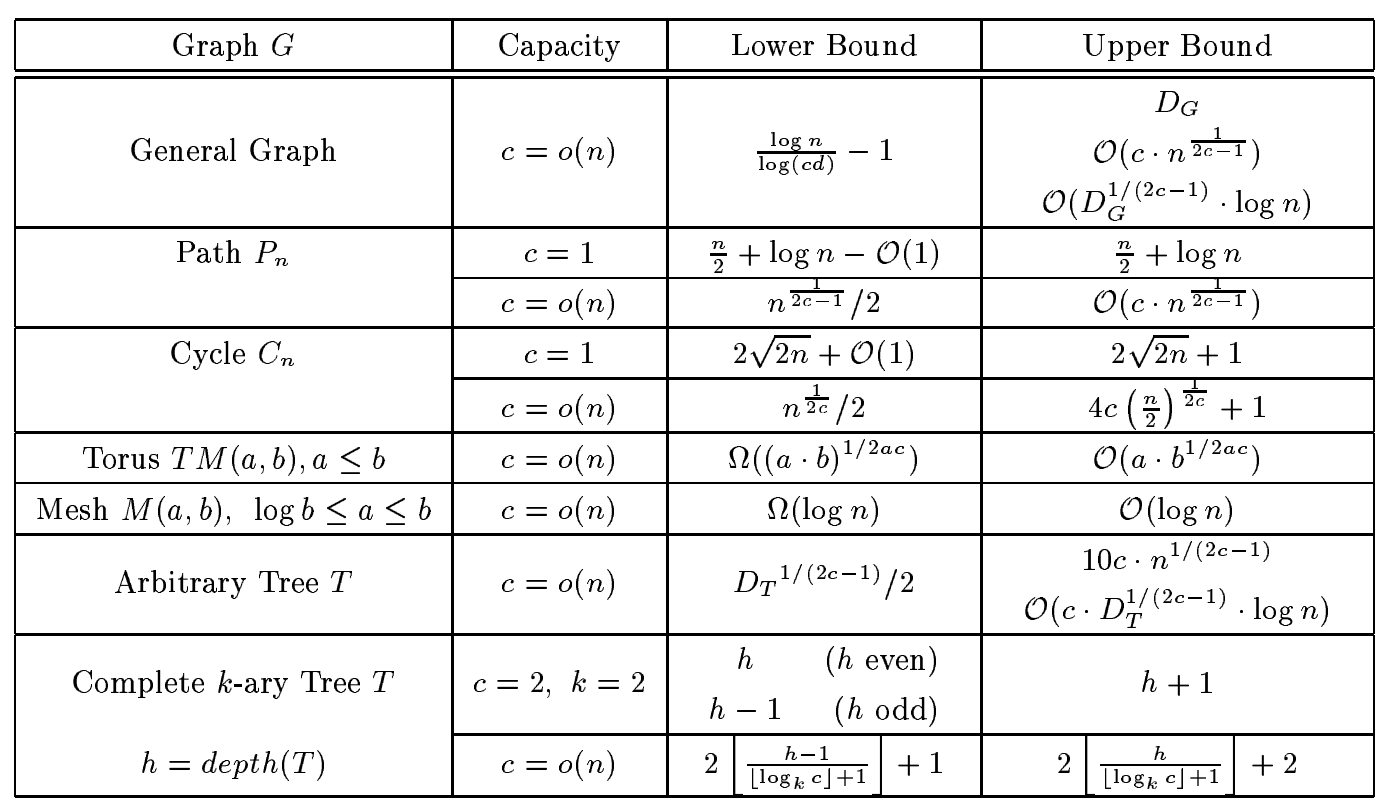

\section{Model}

A physical network is represented by a capacitated digraph $G=(V, E, c)$, that is a directed graph with vertex set $V$ and arc set $E$, together with a positive integral capacity function $c$ on the set of arcs. We always denote by $n$ the number of vertices and in this paper we mostly consider constant capacity functions, i.e. $\forall e \in E, c(e)=c_{0}$.

The network formed by a set of VPs is represented by a digraph $H=\left(V, E^{\prime}\right)$ together with a layout $P$ assigning to each arc $e^{\prime}=(x, y) \in E^{\prime}$ a simple directed path (dipath) $P\left(e^{\prime}\right)$ connecting $x$ to $y$ in $G$. In our terminology, the pair $(H, P)$ is a virtual digraph on $G$, an arc of $H$ is a virtual arc, and the dipath $P\left(e^{\prime}\right)$ in $G$ associated with a virtual arc $e^{\prime}$ is a virtual dipath $(V P)$.

The load of an arc $e$ of $G$ is the number of virtual dipaths containing the arc $e$, that is, $l(e)=\#\left\{e^{\prime} \in E^{\prime} \mid e \in P\left(e^{\prime}\right)\right\}$. A virtual digraph $(H, P)$ satisfying the requirement $\forall e \in E, l(e) \leq c(e)$ is referred to as a c-admissible Directed Virtual Paths Layout of $G$, shortly denoted $c-D V P L$ of $G$. The aim is to design $c$-DVPL of $G$ with minimum hop-count, i.e, to find a virtual digraph with minimum diameter.

For any digraph $F, d_{F}(x, y)$ denotes the distance from $x$ to $y$ in $F$, and $D_{F}$ denotes diameter of $F$. The virtual diameter, $\tilde{D}(G, c)$, of the digraph $G$ with respect to the capacity $c$, is the minimum of $D_{H}$ over all the $c$-DVPL $H$ of $G$. 


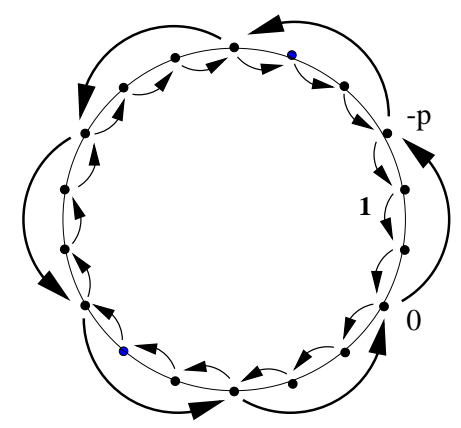

Figure 1: Example of VPL: the cycle, capacity 1

In Fig. 1, $G$ consists of the symmetric directed cycle $C_{n}$. The virtual graph $H$ consists of $\operatorname{arcs}(i, i+1)$ in the clockwise direction and $\operatorname{arcs}(i p,(i-1) p)$ in the opposite direction (assuming that $p$ divides $n$ ). $H$ is in fact a circuit-bracelet (see details in section 3.2). The load of every arc of $C_{n}$ is 1 . Choosing $p=\sqrt{\frac{n}{2}}$ gives good DVPL with diameter at most $2 \sqrt{2 n}+1$.

\section{The Cycle $C_{n}$}

In this section the physical digraph $G$ is $C_{n}$, the symmetric directed cycle of length $n$. We choose arbitrarily a direction on $C_{n}$. For concreteness, consider as positive, or forward (resp., negative or backward) the clockwise (resp., counterclockwise) direction. We assume that $\forall e \in E, c(e)=c^{+}$if $e$ is a forward arc and $c(e)=c^{-}$if $e$ is a backward arc, for some constant nonnegative integers $c^{+}, c^{-}$.

It turns out that our bounds can be expressed as functions of $\sigma=c^{+}+c^{-}$. It is then convenient to define $u b_{C}(n, \sigma)$ (resp., $\left.l b_{C}(n, \sigma)\right)$ as an upper bound (resp., lower bound) on $\tilde{D}\left(C_{n}, c\right)$ valid if $c$ satisfies $c^{+}+c^{-}=\sigma$. By the definition, $l b_{C}(n, \sigma) \leq \tilde{D}\left(C_{n}, c\right) \leq u b_{C}(n, \sigma)$.

\subsection{General Case}

In this section, we show the following upper and lower bounds on the virtual diameter for the cycle.

$$
\frac{n^{\frac{1}{\sigma}}}{2} \leq \tilde{D}\left(C_{n}, c\right) \leq 2 \sigma\left\lceil\left(\frac{n}{2}\right)^{\frac{1}{\sigma}}\right\rceil-2 \sigma+1<2 \sigma\left(\frac{n}{2}\right)^{\frac{1}{\sigma}}+1
$$

The bounds are both proved by induction from the next two lemmas.

Lemma 3.1 $l b_{C}(n, \sigma) \geq \min _{p \in N^{+}}\left\{\max \left(\frac{n}{2 p}, l b_{C}(p, \sigma-1)\right)\right\}$. 
Proof. Let $H$ be an optimal $c$-DVPL of $C_{n}$ and let $\left[x_{1}, y_{1}\right]^{+}$be the dipath consisting of all the vertices of $C_{n}$ between $x_{1}$ and $y_{1}$ in the positive direction. Let $d^{+}\left(x_{1}, y_{1}\right)$ denote the number of arcs in $\left[x_{1}, y_{1}\right]^{+}$. We say that $\left[x_{1}, y_{1}\right]^{+}$is covered by $H$ if (the VP corresponding to) some virtual arc $e^{\prime}$ contains $\left[x_{1}, y_{1}\right]^{+}$. Abusively we say that $\left[x_{1}, y_{1}\right]^{+}$is covered by $e^{\prime}$.
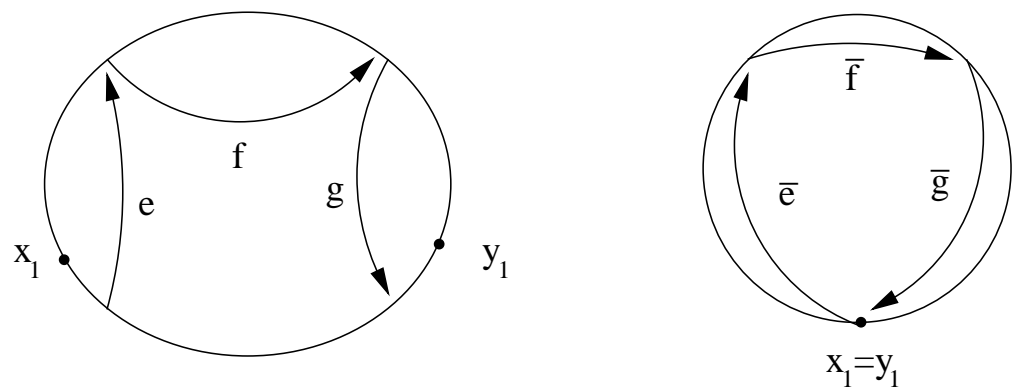

Figure 2: Collapsing a cycle

First we prove that if $\left[x_{1}, y_{1}\right]^{+}$is covered by $e^{\prime}$ then $D_{H} \geq l b_{C}\left(d^{+}\left(x_{1}, y_{1}\right), \sigma-1\right)$. For this, we shorten the cycle by identifying all the nodes in $\left[y_{1}, x_{1}\right]^{+}$with $x_{1}$, obtaining a cycle $C^{\prime}$ of length $d^{+}\left(x_{1}, y_{1}\right)$. Virtual arcs are just transformed according to this graph quotient (see Fig. 2). As example a virtual arc from $x \in\left[x_{1}, y_{1}\right]^{+}$ to $y \in\left[x_{1}, y_{1}\right]^{+}$is left unchanged; and a virtual arc from $x \in\left[x_{1}, y_{1}\right]^{+}$to $y \in\left[y_{1}, x_{1}\right]^{+}$is transformed into the arc $\left(x, x_{1}\right)$. Note that the virtual arc containing the positive arcs of $\left[x_{1}, y_{1}\right]^{+}$is transformed into a loop. We also remove loops or multiple virtual dipaths in order to get a simple DVPL on $C^{\prime}$.

This transformation does not increase the load of any arc; furthermore the virtual arc $e^{\prime}$ that contained $\left[x_{1}, y_{1}\right]^{+}$disappears, so the congestion of any positive arc decreases. Moreover, our transformation does not increase the virtual diameter.

Consequently, we obtain a $c^{\prime}$-DVPL of $C^{\prime}$ (a cycle of length $d^{+}\left(x_{1}, y_{1}\right)$ ) with $c^{\prime+}+c^{\prime-}=\sigma-1$, and diameter at most $D_{H}$. It follows that

$$
D_{H} \geq l b_{C}\left(d^{+}\left(x_{1}, y_{1}\right), \sigma-1\right)
$$

Now we argue that there exist vertices $u$ and $v$ with large $d^{+}(u, v)$ such that $[u, v]^{+}$is covered. Let $\mathcal{P}$ be the shortest dipath in $H$ from 0 to $n / 2$, and assume w.l.o.g. that $\mathcal{P}$ contains the arcs of $[0, n / 2]^{+}$. Let $\mathcal{S}$ denote the set of vertices of $\mathcal{P}$ between $x$ and $y$ in the positive direction. Then $|\mathcal{S}| \leq D_{H}+1$, and therefore there exist vertices $u$ and $v$ such that $[u, v]^{+}$is covered and with

$$
d^{+}(u, v) \geq \frac{n}{2 D_{H}} .
$$

Let $p=\max \left\{d^{+}(u, v) \mid[u, v]^{+}\right.$is covered $\}$. From (2) we have $D_{H} \geq \frac{n}{2 p}$, and from (1) it follows that $D_{H} \geq l b_{C}(p, \sigma-1)$. 
Lemma 3.2 $u b_{C}(n, \sigma) \leq \min _{p \in N^{+}}\left\{2(p-1)+u b_{C}\left(\left\lceil\frac{n}{p}\right\rceil, \sigma-1\right)\right\}$.

Proof. Let us construct a $c$-DVPL on $C_{n}$. Without lost of generality suppose that $c^{+} \geq c^{-}$, so $c^{+} \neq 0$. Let $p \in N^{+}$, we proceed as follows.

- Use $n$ virtual arcs $(i, i+1)_{i \in[0 . . n-1]}$ of dilation 1 in the positive direction.

- Let $S$ be the set of vertices $\left\{0,-p,-2 p, \ldots,-\left(\left\lceil\frac{n}{p}\right\rceil-1\right) p\right\}$, and note that vertices of $S$ form a cycle $C_{\left\lceil\frac{n}{p}\right\rceil}$.

- Use an optimal $c^{\prime}$-DVPL for $C_{\left\lceil\frac{n}{p}\right\rceil}$ with $c^{\prime+}=c^{+}-1$, and $c^{\prime-}=c^{-}$, that is $c^{\prime+}+c^{-}=\sigma-1$.

By construction, the diameter $\Delta(S)$ of the set $S$ (i.e., the maximal distance of two vertices in $S)$ is at most $u b_{C}\left(\left\lceil\frac{n}{p}\right\rceil, \sigma-1\right)$; moreover, for any vertex $x$, we have $d(S, x) \leq p-1$ and $d(x, S) \leq p-1$. Hence $d(x, y) \leq d(S, x)+d(y, S)+\Delta(S) \leq 2(p-1)+u b_{C}\left(\left\lceil\frac{n}{p}\right\rceil, \sigma-1\right)$.

\section{Proposition 3.3}

$$
\frac{n^{\frac{1}{\sigma}}}{2} \leq \tilde{D}\left(C_{n}, c\right) \leq 2 \sigma\left\lceil\left(\frac{n}{2}\right)^{\frac{1}{\sigma}}\right\rceil-2 \sigma+1<2 \sigma\left(\frac{n}{2}\right)^{\frac{1}{\sigma}}+1
$$

Proof. First we consider the lower bound. We prove by induction on $\sigma$ that $l b_{C}(n, \sigma) \geq \frac{1}{2} n \frac{1}{\sigma}$. For the initial case we have $l b_{C}(n, 1)=n-1 \geq \frac{n}{2}$. Now to go from $\sigma-1$ to $\sigma$ we use lemma 3.1 which states that $l b_{C}(n, \sigma) \geq \min _{p \in N+} \max \left(\frac{n}{2 p}, \frac{1}{2} p^{\frac{1}{\sigma-1}}\right)$. An elementary analysis shows that $\max \left(\frac{n}{2 p}, \frac{1}{2} p^{\frac{1}{\sigma-1}}\right) \geq \frac{1}{2} n^{\frac{1}{\sigma}}$ attained for $p=n^{1-1 / \sigma}$. Hence $l b_{C}(n, \sigma) \geq \frac{1}{2} n^{\frac{1}{\sigma}}$ and the proof is completed.

Now, we prove the upper bound. First we show by induction on $\sigma$ that for $n=2 a^{\sigma}, a \in$ $N, u b_{C}(n, \sigma) \leq 2 \sigma\left(\frac{n}{2}\right)^{1 / \sigma}-2 \sigma+1=2 \sigma a-2 \sigma+1$. For $\sigma=1, u b_{C}(n, 1) \leq n-1$ is true. For the inductive step from $\sigma-1$ to $\sigma$, we apply lemma 3.2 with $p=a$, getting $u b_{C}(n, \sigma) \leq$ $2(a-1)+u b_{C}\left(2 a^{\sigma-1}, \sigma-1\right)$. By induction, $u b_{C}\left(2 a^{\sigma-1}, \sigma-1\right) \leq 2(\sigma-1) a-2(\sigma-1)+1$; so we get the expected result.

For other values of $n$, the claim is proved as follows. Let $a=\left[\left(\frac{n}{2}\right)^{1 / \sigma}\right\rceil . a$ is such that $n \leq 2 a^{\sigma}$. As $u b_{C}$ is an increasing function on $n$, we obtain $u b_{C}(n, \sigma) \leq 2 \sigma a-2 \sigma+1=$ $2 \sigma\left\lceil\left(\frac{n}{2}\right)^{1 / \sigma}\right\rceil-2 \sigma+1$. As $a<\left(\frac{n}{2}\right)^{\frac{1}{\sigma}}+1$, this implies $u b_{C}(n, \sigma)<2 \sigma\left(\frac{n}{2}\right)^{\frac{1}{\sigma}}+1$.

In particular we get

Corollary 3.4 If $c^{+}=c^{-}=c$ then

$$
\frac{n^{\frac{1}{2 c}}}{2} \leq \tilde{D}\left(C_{n}, c\right)<4 c\left(\frac{n}{2}\right)^{\frac{1}{2 c}}+1
$$




\subsection{Case $c=1$}

In the case of capacity 1 we have been able to determine $\tilde{D}\left(C_{n}, 1\right)$ up to an additive constant:

$$
2 \sqrt{2 n}-\mathcal{O}(1) \leq \tilde{D}\left(C_{n}, 1\right) \leq 4\left\lceil\sqrt{\frac{n}{2}}\right\rceil-3<2 \sqrt{2 n}+1
$$

The upper bound is the one of proposition 3.3.

Note also that using lemma 3.1 from the starting condition $l b_{C}(n, 2) \geq 2 \sqrt{2 n}+\Theta(1)$ would slightly improve the lower bound on $l b_{C}(n, \sigma)$. The lower bound proof requires some care so we first give some definitions.

Let $H$ be an optimal virtual digraph on $G$ with respect to the capacity 1 . The following definitions are given for the positive direction, but similar notions apply for the negative direction as well.

Definition 3.5 - The forward successor of a vertex $x$ is denoted $x^{+}$,

- $[x, y]^{+}$denotes the dipath from $x$ to $y$ in $C_{n}$ in the positive direction,

- a path $Q=\left(e_{1}^{\prime}, \ldots e_{q}^{\prime}\right)$ from $x$ to $y$ in $H$ is said to be of type + if $[x, y]^{+} \subset W(Q)$. Where $W(Q)$ is the route in $G$ associated to the dipath $Q$ in $H$.

Definition 3.6 $A$ circuit-bracelet of size $n$ is a digraph $A$ of order $n$ constructed as follows (see Fig. 3):

- The digraph is made of a set of cycles $C_{i}, i \in I$ directed in a clockwise manner.

- For any $i, C_{i}$ and $C_{i+1} \bmod I$ share a unique vertex $v_{i+1} \bmod I$.

- The length of the dipath in $C_{i}$ from $v_{i-1}$ to $v_{i}$ is denoted $p_{i}$ and is called the positive length of $C_{i}$; similarly, the length of the dipath in $C_{i}$ from $v_{i}$ to $v_{i-1}$ is denoted $n_{i}$ and is called the negative length of $C_{i}$.

- We denote the successor of $v_{i}$ in $C_{i}$ by $w_{i}$, and the ancestor of $v_{i+1}$ in $C_{i}$ by $z_{i}$.

Let $f(n)$ be the minimal value of $D_{A}$, where $A$ is any circuit-bracelet of size $n$. In the remaining of the section indices are taken modulo $I$.

Lemma 3.7 $f(n)=\tilde{D}\left(C_{n}, 1\right)$

Proof. Notice that if an arc $e$ of $G$ is not used by a virtual dipath $P\left(e^{\prime}\right)$ with $e^{\prime} \in E^{\prime}$, we add a virtual arc $e^{\prime}$ such that $P\left(e^{\prime}\right)=(e)$. This transformation can only decrease the diameter of $H$, which is of no consequence since we only seek for a lower bound on the virtual diameter. Using this manipulation, we know that $\forall e \in E, \exists e^{\prime} \in E^{\prime}$ s.t. $e \in P\left(e^{\prime}\right)$. This implies

$$
\sum_{e^{\prime} \text { arc of type }-} w\left(e^{\prime}\right)=\sum_{e^{\prime} \text { arc of type }+} w\left(e^{\prime}\right)=n .
$$




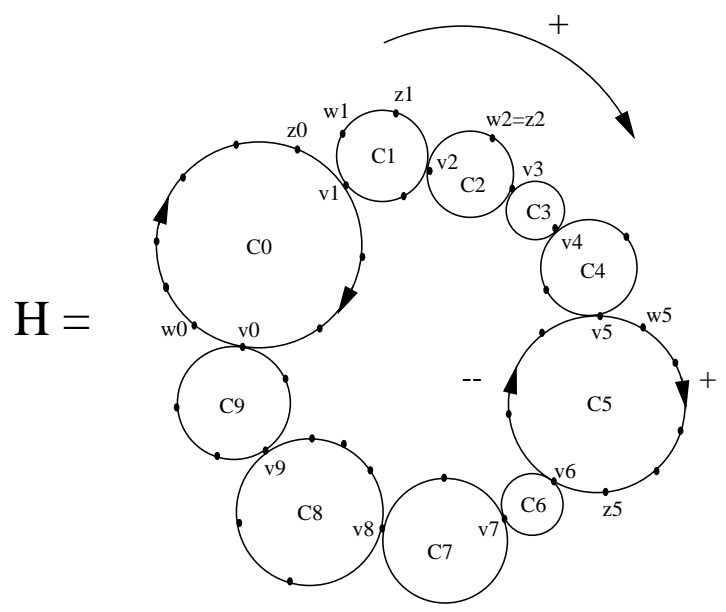

Figure 3: A circuit-bracelet

Where $w\left(e^{\prime}\right)$ is the dilation of a VP $e^{\prime}$, i.e. the length of $P\left(e^{\prime}\right)$.

Now, we show that : If $e^{\prime}=(x, y) \in E^{\prime}$ is an arc of type + of dilation $w\left(e^{\prime}\right) \geq 3$ then all the arcs of type - between $y^{-}$and $x^{+}$are of dilation 1.

Since the capacity of any arc of $G$ is 1 , and there is already a virtual arc of type + between $x$ and $y$, there is no virtual arc of type + ending at any vertex between $x^{+}$and $y^{-}$. Since $H=\left(V, E^{\prime}\right)$ is strongly connected, there is at least one arc ending at each one of these vertices. These arcs are of type - . For the same reasons of capacity and connectivity, these virtual arcs are of dilation 1.

Due to this property it is easy to see that there exists a digraph isomorphism between $H$ and a circuit-bracelet of size $n$ (see Fig. 3).

Lemma 3.8 $f(n)=\Theta(\sqrt{n})$ and the total number of circuits in an optimal circuit-bracelet is also $\Theta(\sqrt{n})$.

Proof. By the construction of lemma 3.2, there exists a regular circuit-bracelet with diameter at most $2 \sqrt{2 n}+1$, so $f(n)=\mathcal{O}(\sqrt{n})$. Note that the size of any circuit in an optimal circuit-bracelet is at most $f(n)+2=O(\sqrt{n})$, otherwise the distance from $w_{i}$ to the second neighbor of $v_{i}$ on the bigger cycle $C_{i}$ is more than $f(n)$. Hence there are at least $\Omega(\sqrt{n})$ circuits. Moreover the total number of circuits is less than $2 f(n)=\mathcal{O}(\sqrt{n})$, otherwise there exist two vertices at distance more than $f(n)$. Thus $f(n)=\Omega(\sqrt{n})$ and the lemma follows. 
We first prove proposition 3.10 for the special case of a regular circuit-bracelet, namely, a circuit-bracelet satisfying $n_{i}=1$ for every $i$. The circuits of a regular circuit-bracelet all consist of a single arc of type - and $p_{i}$ arcs of type + . Remark that $p_{i}$ is then the length of $C_{i}$. Let $g(n)$ denote the minimal value of $D_{A}$ where $A$ is any regular circuit-bracelet of size $n$.

Lemma 3.9 $g(n)=2 \sqrt{2 n}+\mathcal{O}(1)$.

Proof. We prove here that $g(n) \geq 2 \sqrt{2 n}+\mathcal{O}(1)$. We assume that $n$ is sufficiently large. Let $p$ be an integer and $D$ the diameter of the considered circuit-bracelet. Call a circuit big if its size is greater than $\frac{D}{p}$, small otherwise. Recall that the size of any circuit is less than $D+2$. Let $b$ denote the number of big circuits and $s$ denote the number of small circuits. We have

$$
n \leq s \frac{D}{p}+b(D+2) \text { and } s+b \leq 2 D .
$$

Suppose that big circuits are ordered cyclically according to the circuit-bracelet structure: $C_{i_{0}}, C_{i_{1}}, \ldots C_{i_{b-1}}$ as shown on Fig. 3. Let $k \in\{0,1, \ldots b-1\}$ and consider dipaths from $w_{i_{k}}$ to $z_{i_{k+p}}$. In the positive direction the cost is exactly $d_{k}=\sum_{j \in[k, k+p]} p_{i_{j}}-2$; as these circuits are big, $p_{i_{j}} \geq \frac{D}{p}$ and hence $d_{k} \geq \frac{p+1}{p} D-2>D$ if $p<\frac{D}{2}$. So we must use the negative direction. The length is then $d_{k}^{\prime}=p_{i_{k}}+p_{i_{k+p}}+b+s-\left(i_{k+p}-i_{k}\right)-3 \leq D$. Summing on all the $k$ 's we get:

$$
\sum_{k=0}^{k=b-1} d_{k}^{\prime}=2(n-\delta)+b(b+s)-p(b+s)-3 b \leq b D .
$$

Where $\delta$ denote the number of vertices in the small circuits.

So $\frac{2(n-\delta)}{b}+b+s-p-p \frac{s}{b}-3 \leq D$. Note now that $\delta \leq s \frac{D}{p}$, so

$$
\frac{2 n}{b}+b+s\left(1-\frac{2 D}{b p}-\frac{p}{b}\right)-p \leq D
$$

If the coefficient of $s$ in inequality (5) is positive then the left factor of that inequality is greater than $\frac{2 n}{b}+b-p-3$ which is greater than $2 \sqrt{2 n}-p-3$. In turn, the coefficient of $s$ is positive if $b \geq \frac{2 D}{p}+p$.

(4) implies $n \leq 2 \frac{D^{2}}{p}+2 b D$ and so $b \geq \frac{n}{2 D}-\frac{D}{p}$. Using the fact that $D \leq 2 \sqrt{2 n}$, we obtain $b \geq \sqrt{2 n}\left(\frac{p-16}{8 p}\right)$. But

$$
\sqrt{2 n}\left(\frac{p-16}{8 p}\right) \geq \frac{2 D}{p}+p
$$

if $\frac{p}{8}-4-\frac{p^{2}}{\sqrt{2 n}} \geq 0$, and the latter inequality is true if $p \geq 33$ and $n$ is large enough. It follows that $g(n) \geq 2 \sqrt{2 n}-36$. 


\section{Proposition 3.10}

$$
2 \sqrt{2 n}-\mathcal{O}(1) \leq \tilde{D}\left(C_{n}, 1\right) \leq 4\left\lceil\sqrt{\frac{n}{2}}\right\rceil-3<2 \sqrt{2 n}+1
$$

Proof. The upper bound is the one given for the general case. We conjecture that this bound is tight. It would be desirable to obtain a simpler argument that could extend to higher capacities.

Recall that $D=\Theta(\sqrt{n})$. Consider a circuit-bracelet, and recall that $n_{i}+p_{i} \leq D+1$, so that we can find an integer $k$ such that $\sum_{i \in[1, k]}\left(n_{i}+p_{i}\right) \geq 2 D+1$ with $\sum_{i \in[1, k]}\left(n_{i}+p_{i}\right)=\Theta(\sqrt{n})$. Consider the shortest dipath from $v_{1}$ to $v_{k+1}$ and suppose that it uses the positive direction; so $\sum_{i \in[1, k]} p_{i} \leq D$. It follows that $\sum_{i \in[1, k]} n_{i}>D$. So, the dipath from $v_{k}$ to $v_{1}$ cannot use the negative direction, and must use the positive one. It follows that $\sum_{i \notin[1, k]} p_{i} \leq D$. Globally, $\sum p_{i} \leq 2 D=\Theta(\sqrt{n})$. If we remove this $\Theta(\sqrt{n})$ vertices we obtain a regular circuit-bracelet with lesser diameter. It follows that $f(n) \geq g(n-\Theta(\sqrt{n}))=2 \sqrt{2 n} \sqrt{1+\Theta\left(\frac{1}{\sqrt{n}}\right)}=2 \sqrt{2 n}+\Theta(1)$. A new constant appears here in the bound.

\section{The Path $P_{n}$}

In this section the physical digraph $G$ is the $n$-vertex symmetric directed path $P_{n}$. Our bounds are valid for any capacity function $c$ such that positive (resp., negative) arcs have capacity $c^{+}$(resp., $c^{-}$) and the additional requirement $c^{+} \geq 1, c^{-} \geq 1$. Let $\sigma=c^{+}+c^{-}$,

\section{Proposition 4.1}

$$
\frac{n^{\frac{1}{\sigma-1}}}{2} \leq \tilde{D}\left(P_{n}, c\right) \leq 2(\sigma-1)\left\lceil\left(\frac{n-1}{2}\right)^{\frac{1}{\sigma-1}}\right\rceil-2 \sigma+4
$$

Proof. Let us first prove the lower bound. Let $H$ be a $c$-DVPL of $P_{n}$. We say that a sub-path $[x, y]$ is covered by $H$ if the dipaths from $x$ to $y$ and from $y$ to $x$ are both contained in (the VP corresponding to) some virtual arc.

First we show that if $[x, y]$ is covered then $D_{H} \geq l b_{C}(d(x, y), \sigma-2)$. Indeed if $[x, y]$ is covered we identify $x$ and $y$ and collapse the path into a cycle of length $d(x, y)$, we then ignore the virtual paths covering $[x, y]$ (see the proof of lemma 3.1 for details). So doing we obtain a $c^{\prime}$-DVPL for $C_{d(x, y)}$ with $c^{\prime+}+c^{\prime-}=\sigma-2$.

Now, consider two shortest dipaths in $H$, one from 0 to $n-1$ and the second from $n-1$ to 0 . There are at most $2 D_{H}$ intermediate points (including 0 and $n-1$ ) on these two dipaths. Hence we can find two consecutive intermediate vertices $x$ and $y$, with $[x, y]$ covered, such that $d(x, y) \geq \frac{n}{2 D_{H}}$. Thus, if $m=\max \{d(x, y) \mid[x, y]$ is covered $\}$, we have $D_{H} \geq \frac{n}{2 m}$. But due to the covering property $D_{H} \geq l b_{C}(m, \sigma-2)$. Hence 


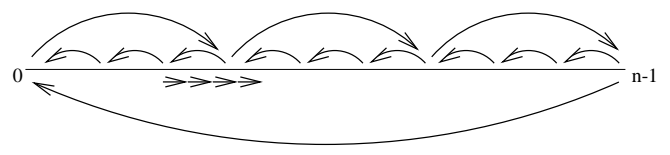

Figure 4: $P_{n}, c=2$

$l b_{P}(m, \sigma) \geq \max \left(\frac{n}{2 m}, l b_{C}(m, \sigma-2)\right)$. Using the lower bound on $l b_{C}(m, \sigma)$ given in proposition 3.3, and maximizing in $m$, completes the lower bound proof.

To prove the upper bound, we construct a VPL based on the best VPL we know on the cycle $C_{n-1}$ with $c^{\prime+}=c^{+}$and $c^{\prime-}=c^{-}-1$. In this VPL, no VP passes over vertex 0 . So, we cut the cycle at vertex 0 and consider it as the path $P_{n}$. On the negative direction, we add a VP of dilation $n$ from $n-1$ to 0 (See Fig. 4). The added VP is used at most once in a path on $H$. The bound is the one for the cycle $C_{n-1}$, sum of capacities $\sigma-1$ plus 1 .

\section{The Complete Symmetric k-ary Tree $T(k, h)$}

In this section the physical digraph $G$ is $T(k, h)$, the directed symmetric complete k-ary tree of depth $h$ rooted at $r_{0}$. Recall that in a complete k-ary tree, each non-leaf vertex has exactly $k$ children. The depth of a vertex is its distance from the root, and the depth of the tree is the maximum depth of any of its vertices. The root $r_{0}$ is the only vertex of in and out-degree $k . T(k, h)$ has $\frac{k^{h+1}-1}{k-1}$ vertices and diameter $2 h$.

The ancestors of a vertex $x$ are all vertices except $x$ on the shortest path connecting $r_{0}$ and $x$. The deepest ancestor of a vertex is its parent, denoted by $f(x)$. A vertex $y$ is said to be below $x$ if $\exists i \geq 0$ s.t. $f^{i}(y)=x$. Note that $x$ is below itself.

\section{Proposition 5.1}

$$
2\left\lfloor\frac{h-1}{\left\lfloor\log _{k} c\right\rfloor+1}\right\rfloor+1 \leq \tilde{D}(T(k, h), c) \leq 2\left\lfloor\frac{h}{\left\lfloor\log _{k} c\right\rfloor+1}\right\rfloor+2
$$

Proof. Let us start with proving the lower bound. Let $H$ be a $c$-DVPL of $T(k, h)$. Let $\gamma=\left\lfloor\log _{k} c\right\rfloor+1$. Let $r$ be a vertex of depth $d, 1 \leq d \leq h-\gamma$; Let $B(\gamma, r)$ denote the complete k-ary subtree of $T(k, h)$ of depth $\gamma$ rooted at $r$. A leaf $x$ of $B(\gamma, r)$ is said to be upward-bad for $r$ if there does not exist any virtual arc $e^{\prime}$ that starts in a vertex below $x$ and ends in a vertex not below $r$. If there doesn't exist any virtual arc $e^{\prime}$ that starts not below $r$ and ends below $x$ then $x$ is said to be downward-bad for $r$. We claim the following: For any vertex $r$ of depth $d, 1 \leq d \leq h-\gamma$ there exist an upward-bad vertex and a downward-bad vertex for $r$. 

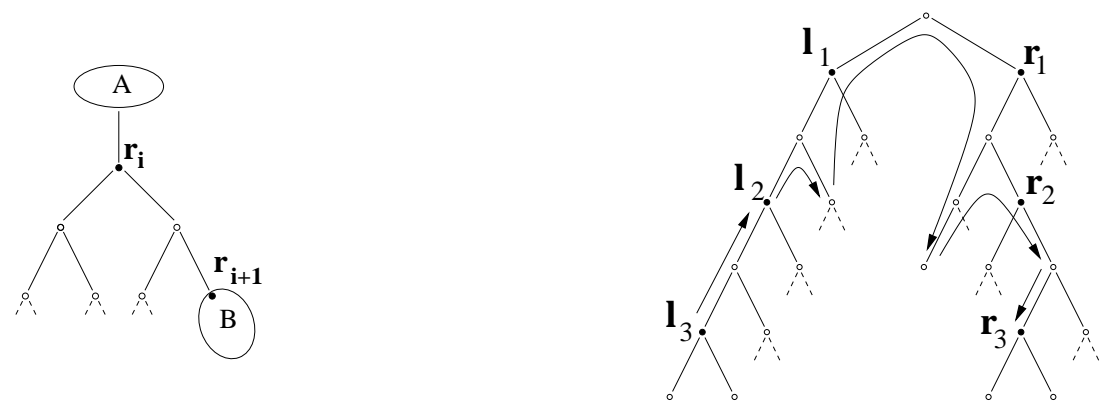

Figure 5: $k=2, c=2$ or $3, \gamma=2$, there exist no $\operatorname{arcs}$ from A to $\mathrm{B}$; (on the right) $k=2$, $\gamma=2, h=6$, one cannot do better than 5 from $l_{3}$ to $r_{3}$

Indeed, suppose that all the $k^{\gamma}$ leaves of $B(\gamma, r)$ are not upward-bad. There exists a virtual arc that starts below each leaf and ends not below $r$. Then the load of the arc $(r, f(r))$ is at least $k^{\gamma}$. Contradicting the fact that the capacity of this arc is $c<k^{\left\lfloor\log _{k} c\right\rfloor+1}$, there exists at least one leaf that is upward-bad for $r$. The same argument considering the load of arc $(f(r), r)$ completes the proof of the claim.

Now we prove that $D_{H} \geq 2\left(\left\lfloor\frac{h-1}{\gamma}\right\rfloor\right)+1$. Let $i_{0}=\lfloor(h-1) / \gamma\rfloor+1$. Define two sequences of vertices $\left(l_{i}\right)_{i=1 . i_{0}}$ and $\left(r_{i}\right)_{i=1 . . i_{0}}$ as follows. Let $l_{1}$ and $r_{1}$ be the leftmost and the rightmost neighbors of $r_{0}$, respectively. If $i \leq i_{0}-1$, choose for $l_{i+1}$ a leaf of $B\left(\gamma, l_{i}\right)$ which is an upward-bad vertex for $l_{i}$. By induction, the depth of $l_{i}$ is $1+\gamma(i-1)$ and if $i \leq i_{0}-1$ it is less than $h-\gamma$ so, from the claim, $l_{i+1}$ exists. Symmetrically, we define the sequence $\left(r_{i}\right)_{i=1 . . i_{0}}$ by choosing $r_{i+1}$ as one of the downward-bad vertices for $r_{i}$.

Let us now consider the shortest path $\mathcal{P}$ in $H$ from $l_{i_{0}}$ to $r_{i_{0}}$. Let $y$ be the first vertex of $\mathcal{P}$ not below $l_{1}$. By construction, $\mathcal{P}$ uses at least $i_{0}$ virtual arcs from $l_{i_{0}}$ to $y$. Also $x$, the predecessor of $y$ in $\mathcal{P}$, is below $l_{1}$ and thus not below $r_{1}$. Hence, $\mathcal{P}$ uses at least $i_{0}$ virtual arcs from $x$ to $r_{i_{0}}$. In summary, $\mathcal{P}$ uses at least $2 i_{0}-1$ virtual arcs. So $D_{G} \geq 2 i_{0}-1$ that is $2(\lfloor(h-1) / \gamma\rfloor)+1$.

To establish the upper bound, we describe a symmetric layout. An example is illustrated in Fig. 6. Each vertex of depth more than $h-\left\lfloor\log _{k} c\right\rfloor$ is linked to all its descendants. The load induced is less than $\frac{k^{\left\lfloor\log _{k} c\right\rfloor}-1}{k-1} \leq c$. Each vertex of depth exactly $h-\left\lfloor\log _{k} c\right\rfloor-t\left(\left\lfloor\log _{k} c\right\rfloor+1\right)$ with $t \geq 0$ is linked to all its ancestors of depth more than $h-\left\lfloor\log _{k} c\right\rfloor-(t+1)\left(\left\lfloor\log _{k} c\right\rfloor+1\right)$. If $h=\left\lfloor\log _{k} c\right\rfloor+t\left(\left\lfloor\log _{k} c\right\rfloor+1\right)+\alpha$ with $t \geq 0$ and $0 \leq \alpha<\left\lfloor\log _{k} c\right\rfloor+1$, the diameter is $2(t+1)$ if $\alpha=0$ and $2(t+2)$ if $\alpha>0$, that is $2\left\lfloor\frac{h}{\left[\log _{k} c\right\rfloor+1}\right\rfloor+2$.

In the special case of $c=2, k=2$ we add two VPs between the two neighbors of the root as shown on Fig. 7. We get an upper bound of $h+1$. 


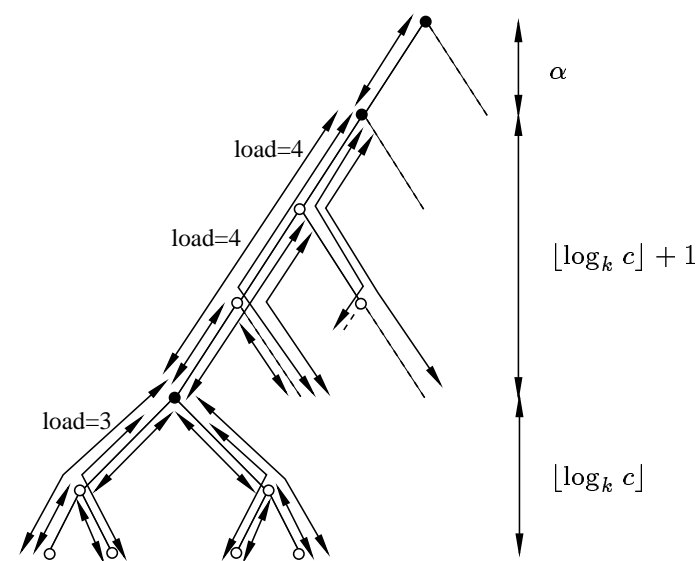

Figure 6: Binary Tree, $c=4, h=6$

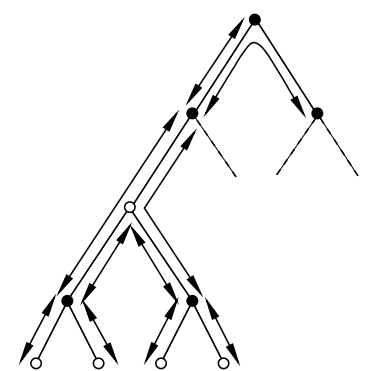

Figure 7: Case $c=2, k=2$

\section{Arbitrary Trees}

In this section the physical graph $G$ is $T$ a tree rooted at $r$. We assume that $\forall e \in E$, $c(e)=c^{+} \geq 1$ if $e$ is an arc going up (from a vertex to its parent) and $c(e)=c^{-} \geq 1$ if $e$ is an arc going down. Again, it turns out that our bound can be expressed as a function of $\sigma=c^{+}+c^{-}$, and therefore it is convenient to define $u b_{T}(\sigma)$ as an upper bound on $\tilde{D}(T, c)$ valid if $c$ satisfies $c^{+}+c^{-}=\sigma$ and $g(\sigma, n)$ an upper bound on $u b_{T}(\sigma)$ valid if $T$ has $n$ vertices. By the definition, $\tilde{D}(T, c) \leq u b_{T}(\sigma) \leq g(\sigma, n)$.

The lower bound follows from our bound for the $n$-vertex path $P_{n}$, upon noting the following. Let $D_{T}$ denote the depth of $T$.

\section{Proposition 6.1}

$$
\tilde{D}(T, c) \geq \tilde{D}\left(P_{D_{T}}, c\right) \geq\left(D_{T}\right)^{\frac{1}{2 c-1}} / 2
$$

Proof. Let $H$ be an admissible virtual graph on $T$. Let $u$ and $v$ be two vertices at maximum distance, $d_{T}(u, v)=D_{T}$. Consider the shortest dipath $\mathcal{Q}$ in $T$ from $u$ to $v$.

We build a VPL on $\mathcal{Q}$ based on $H$. To each VP $P\left(e^{\prime}\right)$ on $T$ is associated a VP on $\mathcal{Q}$ that is the intersection of $P\left(e^{\prime}\right)$ and $\mathcal{Q}$ (i.e. the dipath formed by all the common arcs of $P\left(e^{\prime}\right)$ and $\mathcal{Q})$. Since $T$ is a tree, the intersection of two paths is a well-defined path.

Thus, $H$ induces a VPL on $\mathcal{Q}$ that loads physical arcs no more than the original VPL on $G$. Furthermore the diameter of this new VPL is no more than the diameter of the original one. 


\section{Proposition 6.2}

$$
\tilde{D}(T, c) \leq \tilde{D}\left(C_{2 n-2}, c / 2\right) \leq 2 c(n-1)^{1 / c}+1
$$

Proof. This natural upper bound follows by embedding a cycle around the tree. Consider a cycle $C_{2 n-2}$ embedded around the tree $T$ in a depth-first fashion. Let $c^{+}=\lceil c / 2\rceil$ and $c^{-}=\lfloor c / 2\rfloor$. An admissible graph $H$ on $C_{2 n-2}$ with respect to $c^{+}$on positive arcs and $c^{-}$ on negative ones gives us an admissible virtual graph on $T$.

Our main VPL construction for trees makes use of $\rho$-dominating sets. Hence to establish an upper bound on $\tilde{D}(T, c)$, we need the following two preliminary lemmas regarding the existence of small $\rho$-dominating sets in arbitrary trees.

Definition 6.3 Let $\rho$ be a positive integer. A $\rho$-dominating set is a subset $S$ of $V(T)$ such that for all $x \in V(T), d(x, S) \leq \rho$

Lemma 6.4 For any $\rho$, there exists a $\rho$-dominating set of cardinality at most $\frac{n-1}{\rho+1}+1$.

Proof. Let $L_{i}=\{x \in T ; d(x, r)=i\}$ for $i=0 . . D_{T}$ and $V_{j}=\bigcup_{i=j \bmod (\rho+1)} L_{i} \cup\{r\}$ for $j=0 . . \rho$.

Each $V_{j}$ is a $\rho$-dominating set and the family $\left(V_{j}-\{r\}\right)_{j=0 . . \rho}$ is a partition of $V(T)-\{r\}$. Thus, by choosing the $V_{j}$ of smallest cardinality, we get lemma 6.4.

Lemma 6.5 For any $\rho$, there exists a $\rho$-dominating set $S$ of cardinality at most $2 \frac{n-1}{\rho+1}$ such that the paths from a vertex of $S$ to its deepest ancestor in $S$ are pairwise arc-disjoint.

Proof. Let $S$ be a $\rho$-dominating set of cardinality $s_{0}$. We apply the following algorithm to $S$ :

Initialization $S^{\prime}:=S$.

while there exist two distinct vertices $x$ and $y$ in $S^{\prime}$ such that the deepest common ancestor $z$ of $x$ and $y$ is not in $S$ do

begin

Choose a triple $x, y, z$ such that the depth of $z$ is maximum.

$S^{\prime}:=\left(S^{\prime}-\{x, y\}\right) \cup\{z\}$.

$S:=S \cup\{z\}$.

end

INRIA 
Since the initial set $S$ is a $\rho$-dominating set, the final set $S$, that contains the initial one, is also a $\rho$-dominating set.

Since $r$ can never be deleted from $S^{\prime}$, the cardinality of $S^{\prime}$ must be at least $3(r, x$ and $y$ ) to enter the loop. Thus, since at each step of the algorithm $\left|S^{\prime}\right|$ decreases by 1 , the algorithm terminates at most after $s_{0}-2$ steps. Since at each step $|S|$ increases by 1 , the final set $S$ has cardinality at most $2 s_{0}-2$. By lemma 6.4 if the initial set $S$ is minimal then $s_{0} \leq \frac{n-1}{\rho+1}+1$ and thus the cardinality of the final set $S$ is no more than $2 \frac{n-1}{\rho+1}$.

It remains to prove that paths from a vertex of $S$ to its deepest ancestor in $S$ are pairwise arc-disjoint. Let $t$ and $u$ be two vertices in $S$ such that this condition fails. Let $w$ be their deepest common ancestor. $w$ is not in $S$. We can suppose that there is no vertex of $S$ between $t$ (resp. $u$ ) and $w$. Otherwise, replace $t$ (resp. $u$ ) by the last vertex of $S$ on the dipath from $t$ (resp. $u$ ) to $w$. Since $t$ and $u$ are in the final set $S$, they both have been in some set $S^{\prime}$. Since the algorithm is completed $t$ and $u$ are not both in the final $S^{\prime}$. W.l.o.g we can suppose that $t$ is deleted from $S^{\prime}$ before $u$. Let $i$ be the step of the algorithm where $t$ is deleted from $S^{\prime}$, let $t$ and $y$ be the two vertices chosen by the algorithm at step $i$ and let $z$ is their deepest common ancestor. Since after step $i, z$ is in $S$ and $w$ is below the lowest ancestor of $t$ in the final $S, w$ is strictly below $z$. Since at the end of the algorithm $u$ is in

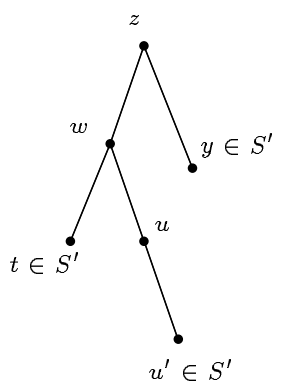

Figure 8: Step $i$ of the algo

$S$, there exists a vertex $u^{\prime}$ in $S^{\prime}$ such that $w$ is the deepest common ancestor of $u^{\prime}$ and $t$. The triple $\left(t, u^{\prime}, w\right)$ should have been chosen instead of $(t, y, z)$ and thus $w$ should be in the final set $S$. This contradicts our hypothesis and completes the proof of lemma 6.5.

\section{Proposition 6.6}

$$
\tilde{D}(T, c) \leq 5 \sigma n^{\frac{1}{\sigma-1}}
$$

Proof. The proof is by induction. We first construct a $\rho$-dominating set $S$ of representative vertices in $T$ using lemma 6.5. Hence every vertex is at distance at most $\rho$ from $S$, and the paths between a vertex of $S$ and its deepest ancestor in $S$ are arc-disjoint. Then we construct 


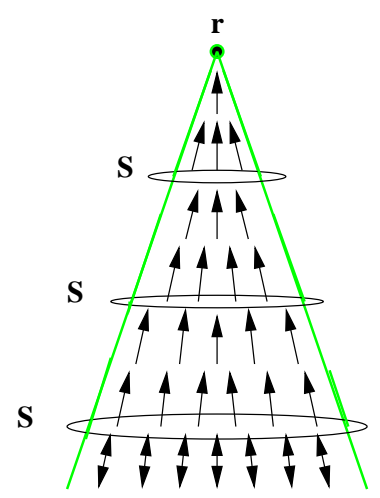

Figure 9: A step in the construction of the VPL. case $1, c^{+} \geq c^{-}$.

a VPL by induction on $\sigma$, i.e., we apply induction on a tree built on the set $S$ with capacity $\sigma-1$.

Given $S$, let $T^{\prime}$ be the tree rooted at $r$ defined by $V\left(T^{\prime}\right)=S$ and

$$
A\left(T^{\prime}\right)=\left\{\left(s, s^{\prime}\right),\left(s^{\prime}, s\right) \mid s^{\prime} \text { is the deepest ancestor of } s \text { in } S\right\} .
$$

An $\operatorname{arc}$ of $T^{\prime}$ corresponds to a path in $T$. By lemma $6.5 \operatorname{arcs}$ of $T^{\prime}$ correspond to arc disjoint paths in $T$ and $\left|V\left(T^{\prime}\right)\right| \leq 2 \frac{|V(T)|-1}{\rho+1}$.

The VPL is built as follow.

If $c^{+} \geq c^{-}$then

All arcs of $\mathrm{T}$ from a vertex to its parent are VPs.

All arcs of $\mathrm{T}$ from a vertex that is not an ancestor of a vertex of $S$ to its child are VPs.

We construct the VPL for the tree $T^{\prime}$ with capacities $c^{++}=c^{+}-1$ and $c^{--}=c^{-}$.

else

All $\operatorname{arcs}$ of $\mathrm{T}$ from a vertex its child are VPs.

All arcs of $\mathrm{T}$ from a vertex that is not an ancestor of a vertex of $S$ to its parent are VPs.

We construct the VPL for the tree $T^{\prime}$ with capacities $c^{+}=c^{+}$and $c^{\prime-}=c^{-}-1$.

To go from a vertex $u$ to a vertex $v$ in the virtual graph, we go from $u$ to a vertex in $S$ in at most $\rho$ steps and then move on the virtual graph built on $T^{\prime}$ (in at most $\tilde{D}\left(T^{\prime}, c^{\prime}\right)$ steps) and then go from a vertex in $S$ to $v$ in at most $\rho$ steps. So, $\tilde{D}(T, c) \leq 2 \rho+\tilde{D}\left(T^{\prime}, c^{\prime}\right)$.

For an arbitrary tree with capacity 1 on each link, the trivial VPL (where each arc becomes a VP) has diameter at most $n$.

INRIA 
$\tilde{D}(T, c) \leq 2 \rho+\tilde{D}\left(T^{\prime}, c^{\prime}\right)$. Thus, $\forall T, \quad \tilde{D}(T, c) \leq 2 \rho+g\left(\sigma-1,2 \frac{n-1}{\rho-1}\right)$ and thus, $g(\sigma, n) \leq 2 \rho+g\left(\sigma-1,2 \frac{n-1}{\rho-1}\right)$.

Let us show by induction that $\forall \epsilon>0, \exists N$ such that $\forall n \geq N, g(\sigma, n) \leq(4+\epsilon) \sigma n^{\frac{1}{\sigma-1}}$. The assertion is true for $c=1$ (i.e. $\sigma=2$ ). Suppose it is true for $\sigma-1$. Then

$$
g(\sigma, n) \leq 2 \rho+(4+\epsilon)(\sigma-1)\left(2 \frac{n-1}{\rho-1}\right)^{\frac{1}{\sigma-2}} .
$$

Let $\rho=2(n-1)^{\frac{1}{\sigma-1}}+1$. Then

$$
\begin{aligned}
g(\sigma, n) & \leq 4(n-1)^{\frac{1}{\sigma-1}}+2+(4+\epsilon)(\sigma-1)(n-1)^{\frac{1}{\sigma-1}} \\
& =(4+\epsilon) \sigma(n-1)^{\frac{1}{\sigma-1}}+2-\epsilon(n-1)^{\frac{1}{\sigma-1}} .
\end{aligned}
$$

For $c=o(n)$ we get $g(\sigma, n) \leq(4+\epsilon) \sigma n^{\frac{1}{\sigma-1}}$ for $n$ greater than some $N$.

Taking $\epsilon=1$ completes the proof of the proposition $\left(N=2^{\sigma-1}+1\right)$.

In particular we have

Corollary 6.7 If $c^{+}=c^{-}=c$ then $\tilde{D}(T, c) \leq 10 \mathrm{cn} \frac{1}{\frac{1}{2 c-1}}$.

Our final construction is given in the following claim.

\section{Proposition 6.8}

$$
\tilde{D}(T, c) \leq \tilde{D}\left(P_{D_{T}}, c\right) \cdot \log n=\mathcal{O}\left(c \cdot D_{T}^{1 /(2 c-1)} \cdot \log n\right)
$$

Proof. Construct an admissible virtual graph $H$ on $T$ by recursively decomposing $T$ using tree separators. A separator node $S(T)$ breaks $T$ into subtrees of cardinality less than $n / 2$. It is well-known that such a separator always exists, and can be found via the following straightforward algorithm. Start with an arbitrary node as $S(T)$. While $S(T)$ does not break the tree $T$ into subtrees of cardinality less than $n / 2$, one of the considered subtrees is of cardinality strictly more than $n / 2$, move $S(T)$ to its neighbor in this subtree.

Let $T_{i}$ be a subtree of $T$ rooted at $r_{i}$ and $v_{i}$ be the only neighbor of $r_{i}$ that is not in $T_{i}$. We describe here a procedure $A\left(T_{i}\right)$, used for constructing $H$.

1. find $S\left(T_{i}\right)$

2. apply the optimal path layout to the path $P_{i}$ from $v_{i}$ to $S\left(T_{i}\right)$.

3. consider the trees $\left(T_{i}^{\prime}\right)_{i=1 . . k}$ made of arcs not already involved in the layout rooted at a neighbor of $S\left(T_{i}\right)$ or of a vertex of $P_{i}$.

4. apply $A\left(T_{i}^{\prime}\right)$ to each subtree $T_{i}^{\prime}$ that is not only one vertex. 


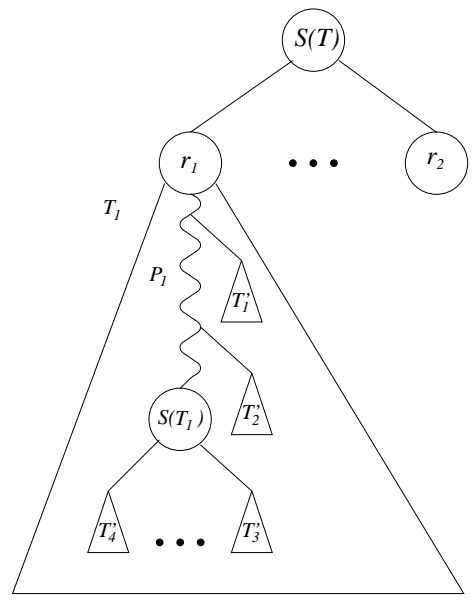

Figure 10:

The construction of $H$ is as follows.

1. find $S(T)$

2. recursively apply $A\left(T_{i}\right)$ on each subtree.

(Note that for the first use of the algorithm, $v_{i}=S(T)$.)

To analyze the diameter of the resulting virtual graph $H$, let

$$
f(n)=\max _{T \text { rooted at } r,|T|=n} \max _{x}\left(d_{H}(r, x), d_{H}(x, r)\right) .
$$

At this point, it is possible to derive a result very similar to that of Proposition 6.6, by observing that $f(n)$ can be bounded recursively by

$$
f(n) \leq f(n / 2)+\tilde{D}\left(P_{n}, c\right) .
$$

This yields that there exists $\alpha(c)$ such that $f(n) \leq \alpha(c) \cdot n^{\frac{1}{2 c-1}}$. Indeed, $\tilde{D}\left(P_{n}, c\right) \leq(4 c-2)\left(\frac{n-1}{2}\right)^{\frac{1}{2 c-1}}+2$ and thus for $\alpha(c) \geq 8 c$ the result is proved by induction. For $c=2$ it gives $f(n) \leq 16 n^{1 / 3}$ and thus $\tilde{D}(T, 2) \leq 32 n^{1 / 3}$.

However, for low-depth trees $T$ it may be preferable to use a different recursive bound for $f(n)$, namely,

$$
f(n) \leq f(n / 2)+\tilde{D}\left(P_{D_{T}}, c\right),
$$

which gives the following upper bound: $f(n) \leq \tilde{D}\left(P_{D_{T}}, c\right) \cdot \log n$. 


\section{Grids and Toroidal Meshes}

In this section we consider as our physical digraph $G$ the toroidal mesh of dimensions $a \times b$, $T M(a, b)$. Recall that $T M(a, b)=C_{a} \square C_{b}$, the Cartesian sum (also called product) of two cycles.

\section{Proposition 7.1}

$$
\tilde{D}(T M(a, b), c) \leq 4(2 c-1) \cdot\left(\frac{a}{2}\right)^{\frac{1}{2 c-1}}+4 a c\left(\frac{b}{2}\right)^{1 / 2 a c}+2 a+1
$$

Proof. $T M(a, b)$ can be viewed as composed of $a$ different main cycles of length $b$ each, numbered from 1 to $a$ and connected to each other cyclically by $b$ transversal cycles numbered from 1 to $b$.

On a cycle of length $b$, the best $a c$-DVPL we have is the one built in section 3. This DVPL is made of $2 a c$ VPs of lengths $\left(\frac{b}{2}\right)^{\frac{i}{2 a c}}$ for $0 \leq i \leq 2 a c-1$. Its diameter is $D_{m} \leq 4 a c\left(\frac{b}{2}\right)^{\frac{1}{2 a c}}+1$.

The VPL we build on $T M(a, b)$ is based on this one. On the $i$-th main cycle (of length $b$ ), we build $2 c$ VPs of lengths

$$
\left(\frac{b}{2}\right)^{\frac{(i-1) c}{2 a c}},\left(\frac{b}{2}\right)^{\frac{(i-1) c+1}{2 a c}}, \ldots\left(\frac{b}{2}\right)^{\frac{i c-1}{2 a c}}
$$

in alternating directions, i.e., one in the positive direction and the next in the negative direction. The load is $c$.

On the $b$ transversal cycles, we build the best DVPL we have with $c^{+}=c$ and $c^{-}=c-1$, as described in section 3.1. The construction sets up $2 c-1$ VPs of lengths

$$
1,\left(\frac{a}{2}\right)^{\frac{1}{2 c-1}}, \ldots\left(\frac{a}{2}\right)^{\frac{2 c-2}{2 c-1}}
$$

again in alternating directions. Hence the load is $c$ in the positive direction and $c-1$ in the negative one. The remaining unit of capacity in the negative direction is used to build VPs of length 1 .

Finally, we have VPs of length 1 in both the directions on the transversal cycles and the virtual diameter of transversal cycles is $D_{t} \leq 2(2 c-1)\left(\frac{a}{2}\right)^{\frac{1}{2 c-1}}+1$.

To move from $[i, j]$ to $\left[i^{\prime}, j^{\prime}\right]$, we first reach the first main cycle where we find main VPs of length $1, \frac{b}{2}, \ldots\left(\frac{b}{2}\right)^{\frac{c-1}{2 a c}}$. It costs at most $D_{t}$ hops to reach $[1, j]$. Then, we move to $\left[1, j^{\prime}\right]$ using main VPs of increasing then decreasing dilation and moving between main cycles using transversal VPs of dilation 1 . It costs at most $2(a-1)+D_{m}$. We then reach $\left[i^{\prime}, j^{\prime}\right]$ in at most $D_{t}$ hops. Finally, we get an upper bound on the virtual diameter of $2 D_{t}+D_{m}+2(a-1)$.

Note that in order to get a graph $G$ such that $\tilde{D}(G, c) \sim \log n$ with $c \geq 1$ we can use a toroidal mesh $T\left(\log n, \frac{n}{\log n}\right)$. Using the lower bound of $\log n$ proved in section 8 we have the following : 
Proposition 7.2 There exists an infinite family of digraphs with $n$ vertices and diameter $n / \log n$, such that $\tilde{D}(G, 1)=\Theta(\log n)$.

For $\left\lceil\log _{2} b\right\rceil \leq a \leq b$ we do not need the toroidal structure to get a good upper bound. We present here a VPL on a mesh $M(a, b)=P_{a} \square P_{b}$ that leads to the following bound.

\section{Proposition 7.3}

$$
\tilde{D}(M(a, b), 1)=\Omega(\log n)
$$

Proof. Construct an admissible virtual graph $H$ on $M(a+1, b+1)$ by patching together a number of strips, defined as follows. Let $A=\left\lceil\log _{2} b\right\rceil, B=\lceil\log (a-A+1)\rceil$, and

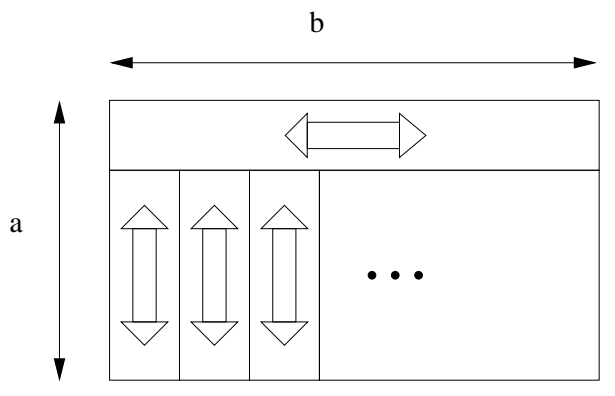

Figure 11: Position of the strips

$C=b+1-\left\lfloor\frac{b+1}{B}\right\rfloor B . H$ is made of a horizontal strip of width $A$, and $\left\lfloor\frac{b+1}{B}\right\rfloor$ vertical strips

of width $B$. (See Fig. 11.) Also, there is a last "remainder" strip of width $C$ put in the opposite direction. A horizontal strip of width $k$ is made of $k$ rows, where row $i, 1 \leq i \leq k$ uses horizontal symmetric arcs of dilation $2^{i-1}$. (See Fig. 12.) A vertical strip is similarly

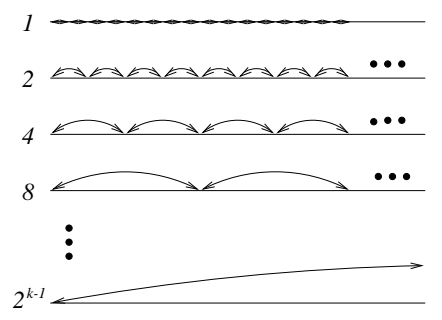

Figure 12: Composition of a strip

defined for vertical virtual arcs. All physical arcs that are not used in virtual arcs involved in the strips are used as virtual arcs of length 1. An example of this construction is depicted in Fig. 13. We next show that the diameter of such a graph is at most $4 A+5 B-C-6$. 


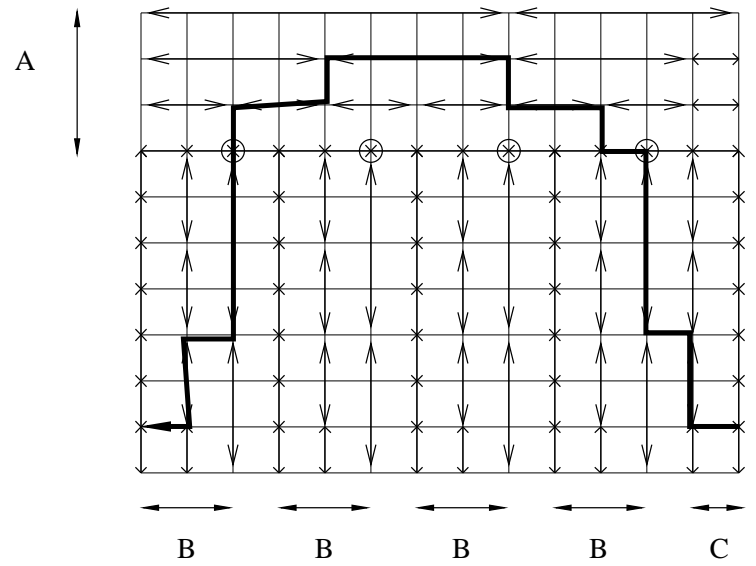

Figure 13: $M(10,13), A=4, B=3, C=2$

Towards that proof, define special vertices named main points. These are the vertices on the $A$ th line from the top, at columns that are multiples of $B$ from the left. The routing strategy is based on reaching a main point from the initial vertex, then reaching the main point closest to the destination vertex using virtual arcs of the horizontal strip, and finally reach the destination point itself. The worst case occurs when the initial vertex is in the last vertical strip (the one of width $C$ ) and the destination point is in a vertical strip of width $B$.

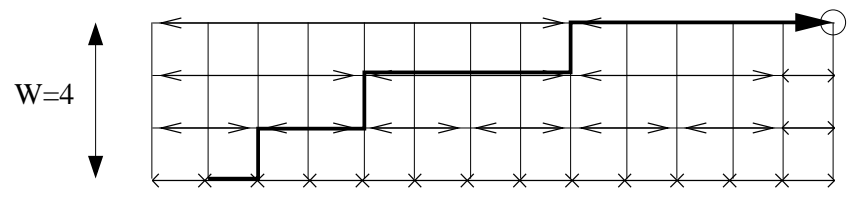

Figure 14: How to move in a strip

As illustrated in Fig. 14, to reach a main point in a strip of width $W$, one needs at most $l(W)=2 W-1$ hops.

The entire route is composed of a number of segments. One needs at most $l(C)$ hops to reach the border of the strip of length $C$, at most $B-C$ hops (using horizontal VPs of length 1 ) to reach the good row in the neighbor strip of length $B$, at most $l(B-C)$ hops to reach a main point, at most $2 l(A)-1$ hops (using VPs of the strip of length $A$ ) to reach the good main point for the destination vertex, and then at most $l(B)$ hops to the destination vertex. Hence the total route is of length at most $4 A+5 B-C-6 \leq 5 \log n$. 


\section{General Bounds}

For most digraphs it turns out that $\tilde{D}\left(G, c_{0}\right)$ is logarithmic even for $c_{0}=1$. Hence the ratio $\frac{\tilde{D}\left(G, c_{0}\right)}{\log n}$ is of importance. For $d$-bounded degree digraphs ${ }^{1}$, a classical result states that $\frac{\log n}{\log \left(c_{0} d\right)}-1 \leq \tilde{D}\left(G, c_{0}\right)$. It is obtained by applying the Moore bound to the virtual digraph with $n$ nodes, degree at most $c_{0} d$, and diameter $\tilde{D}\left(G, c_{0}\right)$ (see $\left.[16,20]\right)$. Note also that $\tilde{D}(G, 1) \leq D_{G}$. Here we derive a tighter bound related to the expansion-congestion parameters of $G$. First we recall three standard definitions. A routing for $G$ is a mapping associating with each ordered pair of vertices $(x, y)$ a route (i.e. a dipath in $G$ ) from $x$ to $y$; the congestion of a routing is the maximal load of an arc of $G$ (i.e., the maximum number of routes going through an arc); the arc-forwarding index of $G$, denoted $\pi(G)$, is the minimum congestion over all possible routings.

The parameter $\pi(G)$ has been extensively studied and many relations exist between $\pi$ and other parameters like bisection or expansion, see [13, 17, 19]. There are strong relationships between $\pi(G)$ and the DVPL issue. A routing for $G$ is a DVPL of $G$ where $H$ is the complete digraph, and so $\pi(G)$ is the smallest integer $c_{0}$ such that $\tilde{D}\left(G, c_{0}\right)=1$.

Proposition 8.1 Let $G$ be a d-bounded digraph.

$$
\frac{\log \pi(G)}{\log \left(c_{0} d\right)}+O\left(\log \tilde{D}\left(G, c_{0}\right)\right) \leq \tilde{D}\left(G, c_{0}\right)
$$

Proof. With every $c_{0}$-DVPL $H$ of $G$ one can associate a routing for $G$ as follows. Note that for any ordered pair of vertices $(x, y)$ there exists at least one dipath in $H$ from $x$ to $y$ with length smaller than $D_{H}$. We select one such dipath and choose the associated real dipath as the route from $x$ to $y$. Due to the capacity constraint, at most $c_{0} d$ virtual dipaths enter (resp., leave) any given vertex of $G$; one can easily check that the number of dipaths in $H$ of length $k$ that use an arc is at most $k c_{0}\left(c_{0} d\right)^{k-1}$. Hence the congestion of our routing is upper-bounded by

$$
M=c_{0}+2 c_{0}\left(c_{0} d\right)+3 c_{0}\left(c_{0} d\right)^{2}+\ldots+D_{H} c_{0}\left(c_{0} d\right)^{D_{H}-1} .
$$

By definition, $\pi \leq M$; as $M \leq c_{0} \frac{D_{H}\left(c_{0} d\right)^{D_{H}}}{c_{0} d-1}$, taking the logarithm we obtain the result.

Remark 8.2 The lower bound of proposition 8.1 is rather similar to the one derived on the gossip time of a network under WDM or wormhole models [7, 2]. In both cases one must construct a route between any pair of vertices: for gossip problems the route is built along $T$ time steps, whereas in the context of VPL design it is constructed by using $\tilde{D}(G, c)$ jumps.

\footnotetext{
${ }^{1}$ where both the in- and out- degrees are upper-bounded by $d$
} 
The following proposition is the counterpart of proposition 7.2, and in some sense establishes its tightness. Specifically, it indicates that for bounded $c_{0}$, one can expect $\tilde{D}\left(G, c_{0}\right)$ to be logarithmic only if $D_{G}$ is not too large. The result is valid for (distance-) symmetric digraphs (namely, such that $d(x, y)=d(y, x)$ ).

Proposition 8.3 Let $G$ be a symmetric bounded degree digraph with $\log D_{G}=\Omega(\log n)$.

$$
\tilde{D}(G, c)=\Theta(\log n) \Longrightarrow c=\Omega\left(D_{G} \log n / n\right)
$$

In particular, if $c$ is constant

$$
\tilde{D}(G, c)=\Theta(\log n) \Longrightarrow D_{G}=O(n / \log n) .
$$

Proof. The idea is that the design of an efficient DVPL is prevented by the existence of a long geodesic dipath contained in $G$. Let us first formalize the notion that a digraph "contains" some bad sub-structure.

Define a retraction of a digraph $G$ as a digraph $G^{\prime}$ such that there exists a mapping $f$ from $V(G)$ onto $V\left(G^{\prime}\right)$ satisfying the following contraction condition: $d_{G}(x, y) \geq d_{G^{\prime}}(f(x), f(y))$.

Define the total load of $G$ for virtual diameter $D_{0}$ as

$\mathcal{L}\left(G, D_{0}\right)=\min \left(\sum_{e \in E} l(e)\right)$,

where the minimum is taken on all DVPL such that $D_{H} \leq D_{0}$.

Due to the contraction condition, for any retraction $G^{\prime}$ of $G$ we have $\mathcal{L}\left(G, D_{0}\right) \geq \mathcal{L}\left(G^{\prime}, D_{0}\right)$. Moreover, denoting the number of arcs of $G$ by $|E|$, the maximum load is greater than or equal to the average load. Hence we have proven the following.

Lemma 8.4 If $G^{\prime}$ is a retraction of $G$ then

$$
\pi\left(G, D_{0}\right) \geq \frac{\mathcal{L}\left(G, D_{0}\right)}{|E|} \geq \frac{\mathcal{L}\left(G^{\prime}, D_{0}\right)}{|E|} .
$$

Next, we claim that the path $P_{D_{G}}$ of length $D_{G}$ is a retraction of $G$. To prove this, consider the following mapping. Label the vertices of $P_{D_{G}}$ by $0,1, \ldots, D_{G}$, and choose a pair of vertices $(x, y)$ of $G$ such that $d(x, y)=d(y, x)=D$; then map any vertex at distance $i$ from $x$ onto vertex $i$ of the path. Due to the triangle inequality, and to symmetry, the mapping is contracting.

Now, suppose that we are given a bounded degree $\operatorname{digraph} G$ with $\log D_{G}=\Theta(\log n)$, and the capacity function $c_{0}$ Consider any DVPL with diameter $D_{H}=\Theta(\log n)$. By lemma 8.4 we have $c_{0} \geq \mathcal{L}\left(L_{D_{G}}, D_{H}\right) /|E|$. We also know that if $D_{0} \sim \log D_{G}$ then $\mathcal{P}\left(L_{D_{G}}, D_{0}\right) \sim D_{G} \log D_{G}$ [21]. It follows that $c_{0} \geq D_{G} \log D_{G} /|E|$. As $|E| \leq n d$, we obtain $c_{0} \geq \frac{D_{G} \log n}{d n}$. 


\section{Open problems and directions}

Some of our bounds are not tight, and the remaining gaps may be narrowed. Establishing upper and lower bounds on $\tilde{D}$ for other families of graphs may also be interesting and useful.

Looking for the minimum diameter is reasonable when all the connections may be requested with roughly the same probability, which is also not always realistic. In case of non-uniform traffic, instead of studying $\tilde{D}$, one may try to optimize its weighted counterpart, $\sum r(i, j) \cdot d_{H}(i, j)$, where $r(i, j)$ denotes the traffic requirements between $i$ and $j$; such a target function may make it desirable to place the VPs between the node pairs which communicate the most.

Finally, there may be other parameters of the directed ATM model worth studying. One may also consider variations on the model with variable capacity of the VPs.

\section{Acknowledgments}

The authors would like to thank Amotz Bar-Noy, Bruno Beauquier, Pascal Chanas, Michele Flammini, Cyril Gavoille and Daniel Kofman for helpful discussions. 


\section{References}

[1] L. Becchetti, P. Bertolazzi, C. Gaibisso, and G. Gambosi. On the design of efficient ATM routing schemes. manuscript, 1997.

[2] J.-C. Bermond, L. Gargano, S. Perennes, A. Rescigno, and U. Vaccaro. Effective collective communication in optical networks. In Proceedings of ICALP 96, volume 1099 of Lectures Notes In Computer Science, pages 574-585. Springer Verlag, July 1996.

[3] M. Burlet, P. Chanas, and O. Goldschmidt. Optimization of VP layout in ATM networks. In preparation, 1998.

[4] P. Chanas. Dimensionnement de réseaux ATM. PhD thesis, CNET Sophia, Sept. 1998. In preparation.

[5] P. Chanas and O. Goldschmidt. Conception de réseau de VP de diamètre minimum pour les réseaux ATM. In Road-f'98, pages 38-40, 1998.

[6] M. De Pricker. Asynchronous Transfer Mode, Solution for Broadband ISDN. Prentice Hall, August 1995. 3rd edition 332p.

[7] O. Delmas and S. Perennes. Circuit-Switched Gossiping in 3-Dimensional Torus Networks. In Proc. Euro-Par'96 Parallel Processing / 2nd Int. EURO-PAR Conference, volume 1123 of Lecture Notes in Computer Science, pages 370-373, Lyon, France, Aug. 1996. Springer Verlag.

[8] T. Eilam, M. Flammini, and S. Zaks. A complete characterization of the path layout construction problem for ATM networks with given hop count and load. In 24th International Colloquium on Automata, Languages and Programming (ICALP), volume 1256 of Lecture Notes in Computer Science, pages 527-537. Springer-Verlag, 1997.

[9] M. Feighlstein and S. Zaks. Duality in chain ATM virtual path layouts. In 4th International Colloquium on Structural Information and Communication Complexity (SIROCCO), Monte Verita, Ascona, Switzerland, July 1997.

[10] O. Gerstel, I. Cidon, and S. Zaks. The layout of virtual paths in ATM networks. IEEE/ACM Transactions on Networking, 4(6):873-884, 1996.

[11] O. Gerstel, A. Wool, and S. Zaks. Optimal layouts on a chain ATM network. In 3rd Annual European Symposium on Algorithms, volume LNCS 979, pages 508-522. Springer Verlag, 1995.

[12] O. Gerstel and S. Zaks. The virtual path layout problem in fast networks. In Symposium on Principles of Distributed Computing (PODC '94), pages 235-243, New York, USA, Aug. 1994. ACM Press. 
[13] M.-C. Heydemann, J.-C. Meyer, and D. Sotteau. On forwarding indices of networks. Discrete Appl. Math., 23:103-123, 1989.

[14] J.-W. Hong, K. Mehlhorn, and A. Rosenberg. Cost trade-offs in graph embedding with applications. J. ACM, 30, 1983.

[15] D. Kofman and M. Gagnaire. Réseaux Haut Débit, réseaux ATM, réseaux locaux et réseaux tout-optiques. InterEditions-Masson, 1998. 2eme édition.

[16] E. Kranakis, D. Krizanc, and A. Pelc. Hop-congestion trade-offs for high-speed networks. International Journal of Foundations of Computer Science, 8:117-126, 1997.

[17] Y. Manoussakis and Z. Tuza. The forwarding index of directed networks. Discrete Appl. Math., 68:279-291, 1996.

[18] A. L. Rosenberg. Issues in the study of graph embeddings. In H. Noltemeier, editor, Proceedings of the International Workshop on Graphtheoretic Concepts in Computer Science, volume 100 of LNCS, pages 150-176, Bad Honnef, FRG, June 1980. Springer.

[19] P. Solé. Expanding and forwarding. Discrete Appl. Math., 58:67-78, 1995.

[20] L. Stacho and I. Vrt'o. Virtual path layouts for some bounded degree networks. In Structure, Information and Communication Complexity, 3rd Colloquium, SIROCCO, pages 269-278. Carleton University Press, 1996.

[21] S. Zaks. Path layout in ATM networks - a survey. In The DIMACS Workshop on Networks in Distributed Computing, DIMACS Center, Rutgers University, Oct. 1997. manuscript. 


\section{Contents}

1 Introduction $\quad 3$

2 Model 5

3 The Cycle $C_{n} \quad 6$

3.1 General Case . . . . . . . . . . . . . . . . . . . . 6

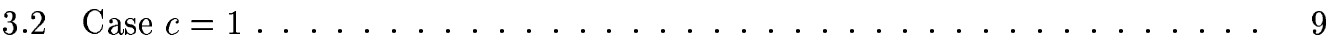

4 The Path $P_{n} \quad 12$

5 The Complete Symmetric k-ary Tree $T(k, h) \quad 13$

6 Arbitrary Trees 15

7 Grids and Toroidal Meshes $\quad 21$

8 General Bounds $\quad 24$

9 Open problems and directions $\quad 26$

$\mathrm{RR} \mathrm{n}^{\circ} 3665$ 


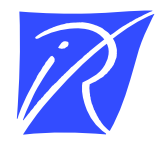

Unité de recherche INRIA Sophia Antipolis 2004, route des Lucioles - B.P. 93 - 06902 Sophia Antipolis Cedex (France)

Unité de recherche INRIA Lorraine : Technopôle de Nancy-Brabois - Campus scientifique 615, rue du Jardin Botanique - B.P. 101 - 54602 Villers lès Nancy Cedex (France)

Unité de recherche INRIA Rennes : IRISA, Campus universitaire de Beaulieu - 35042 Rennes Cedex (France)

Unité de recherche INRIA Rhône-Alpes : 655, avenue de l'Europe - 38330 Montbonnot St Martin (France)

Unité de recherche INRIA Rocquencourt : Domaine de Voluceau - Rocquencourt - B.P. 105 - 78153 Le Chesnay Cedex (France)

Éditeur

INRIA - Domaine de Voluceau - Rocquencourt, B.P. 105 - 78153 Le Chesnay Cedex (France)

http://www.inria.fr

ISSN 0249-6399 